\title{
DESHIDRATACIÓN DE FRUTAS EN UN MÓDULO SOLAR MULTIPROPÓSITO
}

\section{DEHYDRATION OF FRUITS IN A MULTIPURPOSE SOLAR MODULE}

\author{
Julio Oré-García ${ }^{*}{ }^{\circledR}$, Julio Pérez-Sáez ${ }^{2} \mathbb{C}^{-}$, Kléber Janampa-Quispe ${ }^{1}{ }^{\circledR}$, \\ Octavio Cerón-Balboa' ${ }^{1}$, Oswaldo Morales-Morales ${ }^{1}(\mathbb{1}$
}

${ }^{1}$ Facultad de Ingeniería de Minas, Geología y Civil, Universidad Nacional de San Cristóbal de Huamanga, Ayacucho, Perú

${ }^{2}$ Facultad de Ingeniería Química, Universidad Nacional de San Cristóbal de Huamanga, Ayacucho, Perú

Recibido (Recieved): 21/02/2020 Aceptado (Accepted): 05/03/2020

\begin{abstract}
RESUMEN
La ingesta de frutas deshidratadas contribuye a disminuir los problemas digestivos, favorece el tránsito intestinal, mejora el sistema gastrointestinal y previene los problemas de garganta, entre otras bondades. En este trabajo, utilizando un módulo solar multipropósito (MSM), el cual fue diseñado y construido por el equipo investigador del Centro de Energías Renovables de la Universidad Nacional de San Cristóbal de Huamanga (UNSCH), se deshidrató manzanas, piñas y plátanos, constituyendo un producto a comercializar, al cual lo denominamos SNACK SOLAR. La evaluación físico-químico de estas frutas deshidratadas muestran que se mejoró enormemente la concentración del ácido ascórbico (vitamina C), así en 100 g de manzana aumentó de $0.833 \mathrm{mg}$ a $13.33 \mathrm{mg}$, en $100 \mathrm{~g}$ de piña aumentó de $27.5 \mathrm{mg}$ a $39.33 \mathrm{mg}$ y en 100 g de plátanos aumento de $13.83 \mathrm{mg}$ a $39.33 \mathrm{mg}$, lo cual representa un aporte significativo a la alimentación humana, más aún, utilizando como recurso la energía solar térmica. El snack solar no lleva ningún preservante, ni saborizante, como si lo llevan los productos similares industrializados, por ello también, su tiempo de vida es de solo algunos días. Este proyecto es una propuesta innovadora de incubadora de micro empresas que surge desde el interior de las aulas universitarias.
\end{abstract}

Palabras Clave: energía solar térmica, deshidratación de frutas, micro empresas innovadoras.

\section{ABSTRACT}

The intake of dehydrated fruits helps to reduce digestive problems, promotes intestinal transit, improves the gastrointestinal system and prevents throat problems, among other benefits. In this work, using a multipurpose solar module (MSM), which was designed and built by the research team of the Center for Renewable Energies of the National University of San Cristóbal de Huamanga (UNSCH), dehydrated apples, pineapples and bananas, constituting a product to commercialize, which we call SNACK SOLAR. The physical-chemical evaluation of these dehydrated fruits shows that the concentration of ascorbic acid (vitamin C) was greatly improved, thus in $100 \mathrm{~g}$ of affected apple from $0.833 \mathrm{mg}$ to $13.33 \mathrm{mg}$, in 100 g of affected pineapple from $27.5 \mathrm{mg}$ to $39.33 \mathrm{mg}$ and in $100 \mathrm{~g}$ of bananas increased from $13.83 \mathrm{mg}$ to $39.33 \mathrm{mg}$, which represents a significant contribution to human food, even more, using thermal solar energy as a resource. The solar snack does not carry any preservatives or flavoring, as if they carry similar industrialized products, so also, their life time is only a few days. This project is an innovative proposal of micro business incubator that arises from inside the university classrooms.

Keywords: solar thermal energy, fruit dehydration, innovative micro companies.

\footnotetext{
* Corresponding author.:

E-mail: julio.ore@unsch.edu.pe
} 


\section{INTRODUCCIÓN}

La energía es la base de toda actividad humana, por lo que debe ser utilizada con eficiencia $y$ responsabilidad ambiental. El Centro de Conservación de Energía y del Ambiente del Perú ha realizado un diagnóstico de la situación actual del uso de la energía solar y eólica en el país [1]; en ella se muestra que Ayacucho, siendo un departamento donde la energía solar es rentable, sin embargo, existe una escasa cantidad de unidades instaladas tanto en las aplicaciones térmicas como fotovoltaicas. De esta escasa cantidad, es el sector familiar el que dispone de la menor capacidad instalada.

La energía del sol tiene una ventaja con respeto a otras fuentes. "Se pueden construir dispositivos solares de cualquier tamaño y por ser de tipo modular también se pueden ampliar" [2].

Actualmente, existen diversas alternativas para realizar el proceso de deshidratación de alimentos, dentro de las cuales se considera el deshidratador solar. Los equipos empleados para este fin presentan mayor eficiencia que el secado directo al sol y menores costos de operación con respecto a los sistemas eléctricos convencionales.

El consumo de frutas deshidratadas es una gran fuente de nutrientes, puesto que el contenido de agua de las frutas se reduce, y aumenta la concentración de sus nutrientes, especialmente en fibra y carbohidratos. El análisis de la vitamina $C$ es un micronutriente esencial en la alimentación del hombre, y es considerado la vitamina más sensible al someterlo a temperaturas altas y si no se controla el proceso se pierde hasta el $100 \%$ de su concentración [3].

En este trabajo utilizando un secador solar multipropósito, el cual fue diseñado y construido en el Centro de Energías Renovables de la Universidad Nacional de San Cristóbal de Huamanga, se deshidrató manzanas, plátanos y piña. De las frutas secas obtenidas, se midió la concentración de ácido ascórbico, azúcar y humedad, a fin de determinar la calidad del producto. Finalmente se envasó herméticamente en envases de plásticos, para comercializarlos dentro de la ciudad universitaria de la UNSCH.

\section{ANTECEDENTES}

El secado de productos agrícolas, hierbas, frutas, etc., es un método de conservación de alimentos utilizado desde el Neolítico, utilizados con fines de la conservación, reducir el peso en el transporte y mejorar la calidad del producto final. En la actualidad el secado se emplea comercialmente en más de 50000 tipos de productos diferentes, a velocidades que varían entre unos pocos gramos por hora hasta toneladas por hora, en tiempos que oscilan entre pocos segundos a meses. Para ello, en la literatura se han reportado más de 500 tipos diferentes de secaderos de toda clase [4].

El secado de frutas con tecnologías solares para fines comerciales no son muy difundidos en el mercado local, ni nacional, esto motiva a la realización de este proyecto, que surge como una idea de negocios desde las aulas universitarias.

\section{METODOLOGÍA}

\subsection{MATERIALES}

- Módulo Solar Multipropósito (MSM).

- Moisture Analyzer. AND MX-50. 0,01 \% / Max. 51 g. H.W. Kessel S.A.

- Refractómetro de mesa marca ABBE.

- Materiales para la evaluación de las frutas secas:

- Probetas

- Pipetas

- Vasos de precipitado de $400 \mathrm{ml}$

- Erlenmeyers de $250 \mathrm{ml}$

- Buretas de $10 \mathrm{ml}$

- Balanza

- Licuadora

- Fiolas

- Cuchillos

- Reactivos:

- 2-6 Diclorofenolindofenol (DFIF)

- Ácido oxálico

- Ácido ascórbico

- Muestras:

- Piña (Ananas comosus) var. Golden

- Manzana (Malus domestica) var. Delicias

- Plátano de freir (Musa balbisiana).

\subsection{MÉTODOS}

\subsubsection{MÉTODOS DE ANÁLISIS FíSICO QUÍMICO}

\subsubsection{EVALUACIÓN DEL ÁCIDO ASCÓRBICO}

Se aplicó el método 967.21, 45.1.14, de la AOAC [5], siguiendo los siguientes pasos:

\section{a) Preparación de estándares:}

Ácido oxálico al $0.5 \%$

Preparar 1 I de solución de ácido oxálico al 0.5\%

Ácido ascórbico en ácido oxálico

Disolver $100 \mathrm{mg}$ de ácido ascórbico en $100 \mathrm{ml}$ de solución de ácido oxálico al $0.5 \%$ en una fiol de $100 \mathrm{ml}$. Esta solución, que contiene $0.1 \%$ de ácido ascórbico que es muy inestable y se prepara el mismo momento de la determinación.

2-6 Diclorofenolindofenol 
Disolver $100 \mathrm{mg}$ de 2-6 diclorofenolindofenol en 100 $\mathrm{ml}$ de agua destilada hirviente. Enrasar a $100 \mathrm{ml}$ cuando esté fría. Almacenar en botella oscura y en refrigeración.

b) Cálculo del equivalente en ácido ascórbico por ml de solución de 2-6-DFIF:

Tomar $1 \mathrm{ml}$ de la solución de ácido ascórbico en ácido oxálico y colocar en erlenmeyer de $50 \mathrm{ml}$.

Agregar $30 \mathrm{ml}$ de solución de ácido oxálico al $0.5 \%$

Titular con solución de 2-6-DFIF, la cual estará contenida en una microbureta de $10 \mathrm{ml}$. El final de la titulación es indicado por el cambio a color rosado débil por 10 - 15 s. La solución de 2-6-DFIF debe estandarizarse cada día.

Eq. = mg de ácido ascórbico/ $\mathrm{ml}$ de solución de 2-6 DFIF gastados en la titulación

Se determina en $0,33 \mathrm{mg} / \mathrm{ml}$ el equivalente en ácido ascórbico por $\mathrm{ml}$ de solución de 2-6- DFIF

\subsubsection{DETERMINACIÓN CONCENTRACIÓN DE AZÚCAR}

DE

LA

Se pesan $5 \mathrm{~g}$ de muestras y se introducen en un vaso de precipitado con $25 \mathrm{ml}$ de agua destilada.

Se deja reposar por 60 minutos, se tritura y se agita durante 20 minutos a fin de extraer los sólidos solubles.

Se mide la concentración de sólidos solubles con la ayuda del refractómetro a $20^{\circ} \mathrm{C}$.

\subsubsection{EVALUACIÓN DEL PORCENTAJE DE HUMEDAD}

La determinación de humedad se desarrolló colocando entre 2 y $3 \mathrm{~g}$ de muestra en el determinador de humedad (Moisture Analyzer) y se programó el equipo para obtener el porcentaje de humedad. El procedimiento se desarrolló tanto para las muestras frescas como muestras secas.

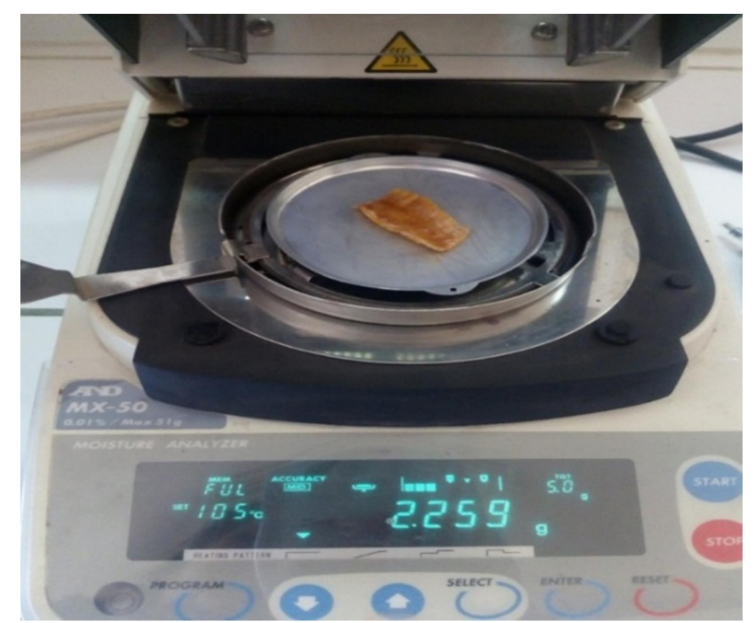

Fig. 1. Evaluación del secado de los productos del snack solar

\subsubsection{PROCEDIMIENTO PARA OBTENER EL SNACK SOLAR}

a) Obtención de la materia prima, se realizó en el mercado de frutas, siempre con la garantía de que las frutas estén en buen estado.

b) Selección de la materia prima, la selección es un proceso importante ya que depende de ésta el buen resultado del producto final; se tiene que seleccionar plátanos de freír maduros, las piñas tienen que estar en estado "pintón" (no tan maduras ni verdes), el tipo golden son las más agradables en sabor y olor. Las manzanas tienen que ser de un tamaño medio.

c) Lavado y desinfección de la materia prima, Se lavaron las frutas con agua corriente y se sumergieron en solución de hipoclorito de sodio con una concentración de cloro de 70 ppm durante 3 minutos.

d) Pelado de la materia prima, se pelaron los plátanos y las piñas. Las manzanas no se pelan, para mantener su textura

e) Laminado de la materia prima, se cortaron láminas de 2 a $3 \mathrm{~mm}$ de espesor (fig. 2) buscando la mayor uniformidad de los cortes para que el proceso de secado sea efectivo, luego se colocaron en las bandejas de secado del MSM.

f) Introducción de la materia prima al MSM, se colocaron las bandejas con las frutas dentro del MSM y se aseguró las compuertas de entrada y salida de aire, a fin de garantizar el flujo de aire adecuado (fig. 3)

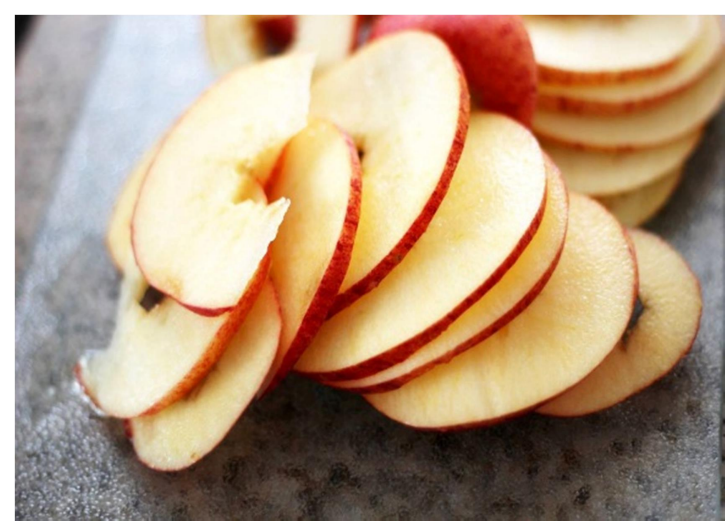

Fig. 2. Rebanado de las frutas

g) Orientación del MSM, cada hora se orientó el MSM de modo tal que la radiación solar incida sobre la placa de vidrio, también se fue variando la inclinación del reflector a fin de direccionar los rayos solares a la placa colectora. . La curva de deshidratación de cada

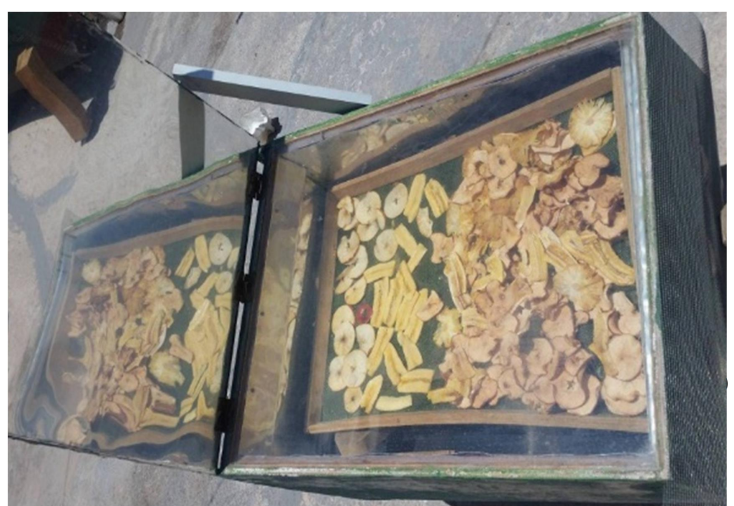


fruta elegida se desarrolló determinando la pérdida de agua con respecto al tiempo, utilizando el Moisture Analyzer AND MX-50.

Fig. 3. Secado de los productos del Snack Solar en el MSM

h) Enfriamiento del producto, pasado entre uno a dos días dependiendo del producto, se retiró la bandeja con las frutas y se dejó enfriar por unos 30 minutos. Cada fruta seca fue analizada en los parámetros predeterminados.

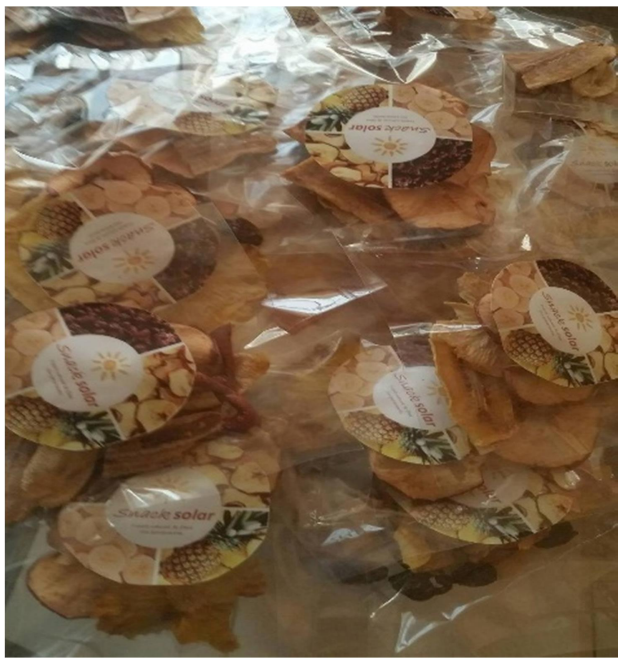

a) i) Envasado del producto, para asegurar su conservación el envasado se realizó en bolsas de aluminio y/o polietileno de alta densidad, selladas. Para una mejor presentación y cumplir con los requisitos de un snack, se envasaron las frutas secas mezcladas y se le añadió algunas pasas secas. Se puso una etiqueta con la información nutricional básica.

j) Comercialización del snack solar, se pasó por dos presentaciones como se muestra en la figura 4, para finalmente comercializarlos dentro de la comunidad universitaria.

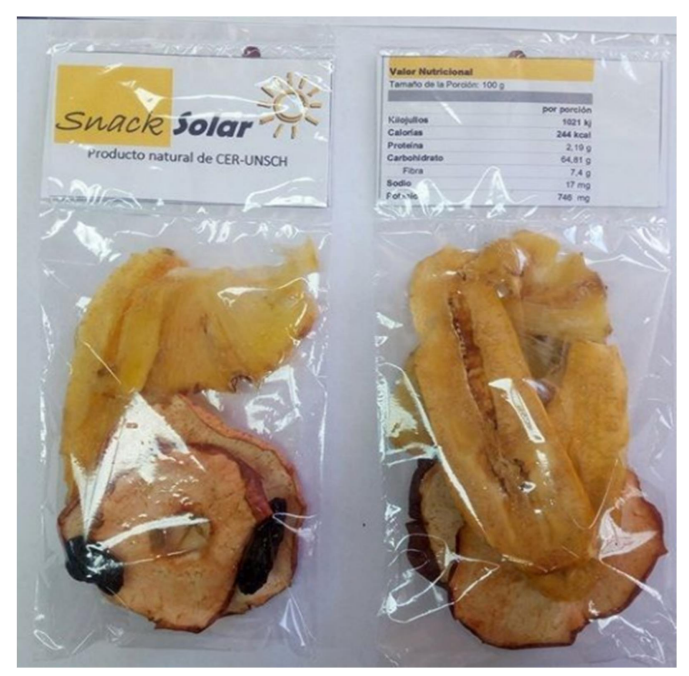

b)

Fig. 4. Presentación. (a) Inicial y (b) actual del snack solar.

\section{ANÁLISIS DE RESULTADOS}

\subsection{PARÁMETROS TÉRMICOS DEL MÓdULO SOLAR MULTIPROPÓSITO (MSM)}

El secador del MSM (fig. 5) es un secador pasivo de cabina, como sistema pasivo utiliza la circulación natural del aire y dispone de un captador solar de placa plana [6].

Sin carga, la temperatura del aire alcanza $74^{\circ} \mathrm{C}$ manteniendo una diferencia de $40^{\circ} \mathrm{C}$ respecto a la temperatura de entrada del aire, valor óptimo requerido para secado [7]. De acuerdo a S. Nandwani [8] cuando la temperatura del gabinete de un secador alcanza valores entre $50^{\circ} \mathrm{C}$ y $70^{\circ} \mathrm{C}$ en ella se pueden secar casi todos los productos a baja escala, es decir para un uso doméstico, como es la orientación del MSM.

La temperatura de calentamiento del aire a la salida de la cámara de secado es de $74^{\circ} \mathrm{C}$ para una radiación solar promedio $960 \mathrm{~W} / \mathrm{m} 2$ de y temperatura ambiente de $31^{\circ} \mathrm{C}$ y velocidad de viento de $0,8 \mathrm{~m} / \mathrm{s}$.

La temperatura de la placa alcanza un valor de $173^{\circ} \mathrm{C}$, y permanece estacionaria para una intensidad de radiación solar de $774 \mathrm{~W} / \mathrm{m} 2$.
El primer factor de mérito $\mathrm{F} 1$ es de 0,001 \pm 0,001, mientras el segundo factor de mérito F2 es de 0,41.

\subsection{EVALUACIÓN FÍSICO QUÍMICA DE LOS PRODUCTOS DESHIDRATADOS}

\subsubsection{DETERMINACIÓN DE HUMEDAD}

La humedad inicial de las frutas utilizadas fueron:

$$
\begin{array}{lll}
\text { - } & \text { Manzana } & : 83 \% \\
\text { - } & \text { Piña } & : 80 \% \\
\text { - } & \text { Plátano } & : 68 \%
\end{array}
$$

Las humedades son cercanas a las de productos similares que se presentan en las tablas Peruanas de Composición de los Alimentos [9], que señalan $84.7 \%$ para la manzana; $89.3 \%$ para la piña y $68.1 \%$ para el plátano para freír maduro.

Las frutas secas tuvieron las siguientes humedades:

$\begin{array}{lll}\text { - } & \text { Manzana } & : 14 \% \\ \text { - } & \text { Piña } & : 12 \% \\ \text { - } & \text { Plátano } & : 15 \%\end{array}$




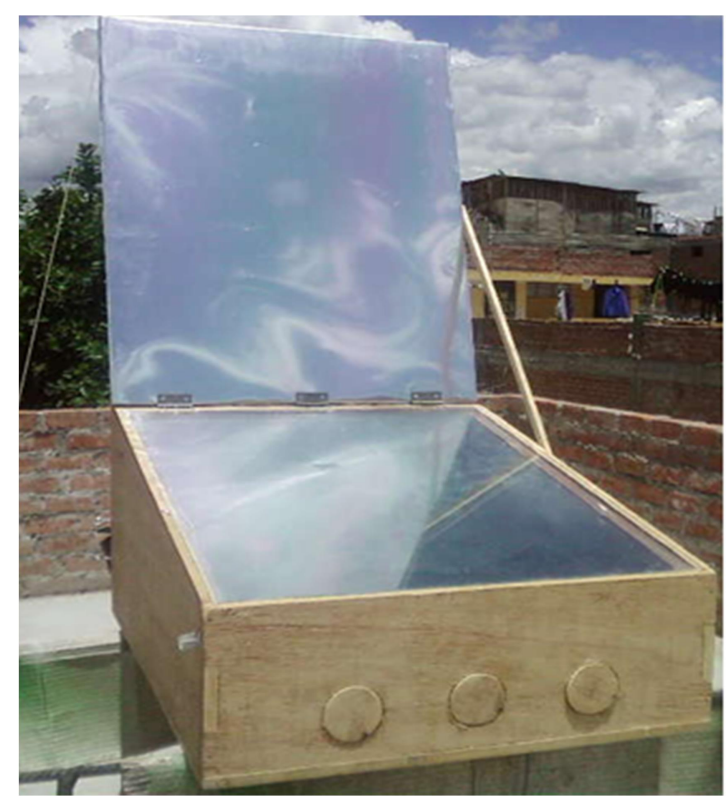

Fig. 5. El Módulo Solar Multipropósito.

Las humedades finales alcanzadas están por debajo de $15 \%$. De acuerdo a Baduí [10], una humedad superior a $15 \%$ permite el crecimiento de hongos, por lo que los alimentos secos tienen este contenido de humedad como el límite máximo permisible para una conservación de mediano o largo plazo. Nuestros productos se pueden conservar por tiempos mucho mayores que los productos frescos, sin necesidad de adición de conservantes.

\subsubsection{CONTENIDO DE VITAMINA C}

El contenido de vitamina C para la manzana fresca fue de $0.833 \mathrm{mg} / 100 \mathrm{~g}$, que resulta algo menor que el señalado en las tablas del INS (2009) que es de 1.3 $\mathrm{mg} / 100 \mathrm{~g}$ para la manzana nacional. La diferencia se debe probablemente a que se trata de diferentes variedades. En el caso de la piña, nuestro resultado fue de $27.5 \mathrm{mg} / 100 \mathrm{~g}$, mayor a los $19.9 \mathrm{mg} / 100 \mathrm{~g}$ de las tablas del INS. Finalmente, para el plátano fue de 13.83 $\mathrm{mg} / 100 \mathrm{~g}$, algo mayor a los $10,40 \mathrm{mg} / 100 \mathrm{~g}$ de las tablas referidas. El proceso de secado provoca un incremento en la concentración de ácido ascórbico por la eliminación de gran cantidad de agua. Los contenidos de humedad final de los productos secos fueron: Manzana $8.16 \mathrm{mg} / 100 \mathrm{~g}$, piña $39.33 \mathrm{mg} / 100$ g y plátano $32.15 \mathrm{mg} / 100 \mathrm{~g}$.

\subsubsection{CONTENIDO DE SÓLIDOS SOLUBLES}

El contenido de azúcares de los productos secos fue de 25 \% tanto para la manzana como para la piña y de $14,29 \%$ para el plátano.

La tabla I resume los resultados de la evaluación de las propiedades de las frutas secas y de la velocidad de secado en cada caso.

TABLA I

Resumen de la evaluación físico-química de los productos del Snack Solar

\begin{tabular}{ccccc}
\hline Muestra & Humedad eliminada & $\begin{array}{c}\text { Porcentaje de azúcar } \\
\text { (refractómetro) }\end{array}$ & $\begin{array}{c}\text { Ácido ascórbico en } \\
100 \text { g fruto fresco }\end{array}$ & $\begin{array}{c}\text { Ácido ascórbico en } \\
100 \text { g fruto seco }\end{array}$ \\
\hline Manzana & $7.04 \%$ en $13.3 \mathrm{~min}$ & $25 \%$ & $0.833 \mathrm{mg}$ & $8.16 \mathrm{mg}$ \\
Piña & $4.18 \%$ en $17.6 \mathrm{~min}$ & $25 \%$ & $27.5 \mathrm{mg}$ & $39.33 \mathrm{mg}$ \\
Plátano & $3.49 \%$ en $29.1 \mathrm{~min}$ & $14.3 \%$ & $13.83 \mathrm{mg}$ & $32.15 \mathrm{mg}$ \\
\hline
\end{tabular}

Nota: Elaboración propia

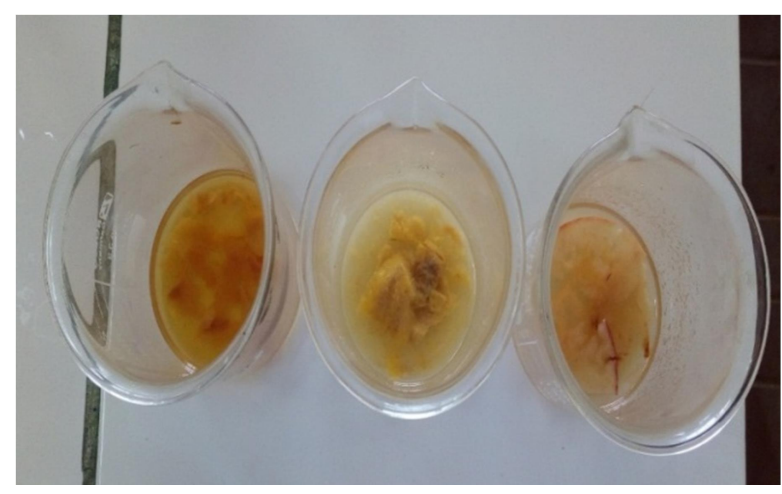

a)

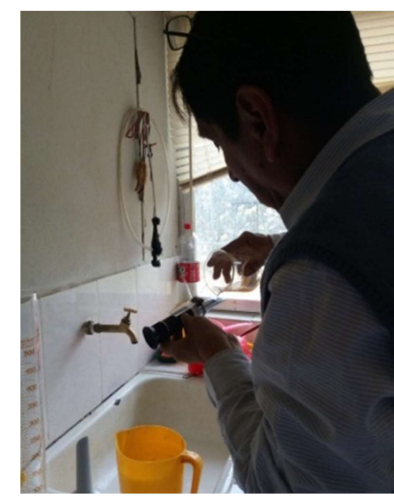

b)

Fig. 6. Análisis de la concentración de azúcar en los productos del snack solar, (a) preparación de muestra y (b) disposición en el refractómetro. 


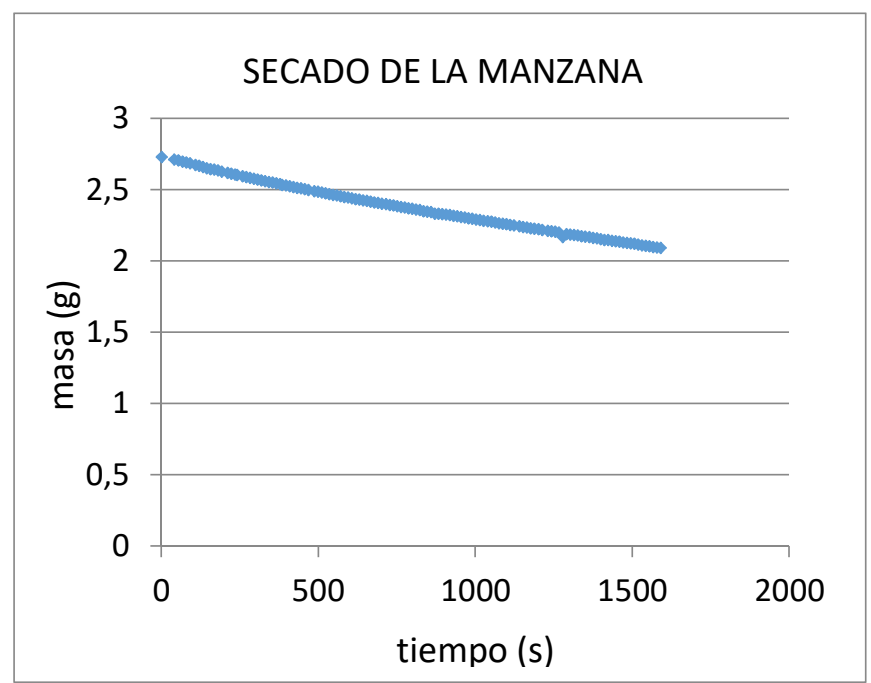

a)

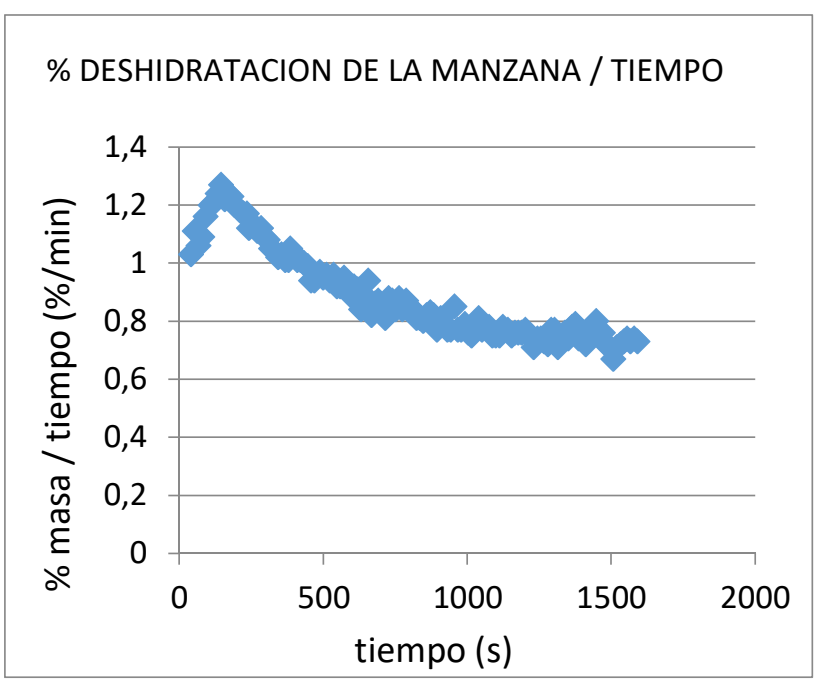

b)

Fig. 7. Deshidratación de la manzana, (a) curva de secado y (b) rapidez de deshidratación porcentual.

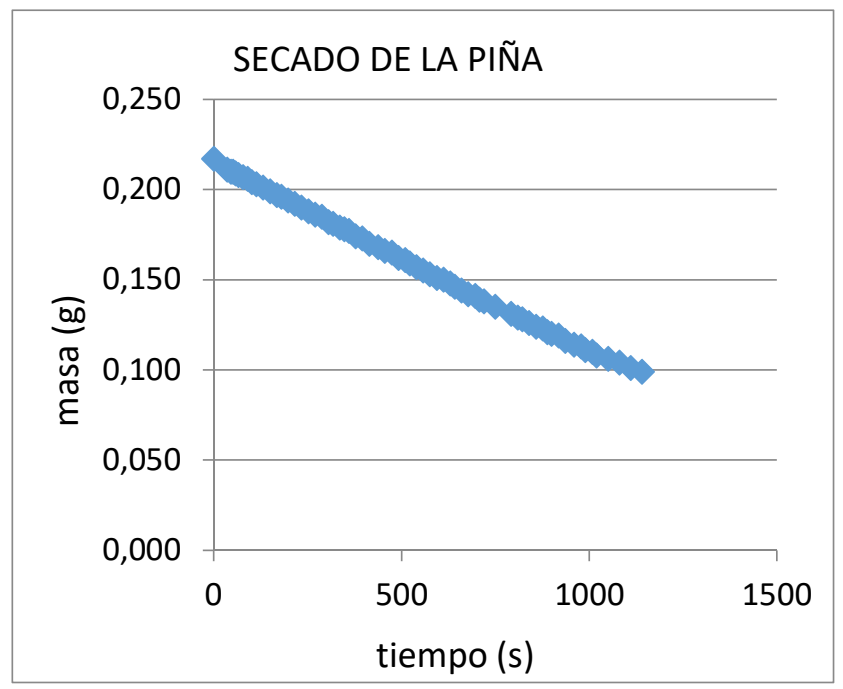

a)

Fig. 8. (a) Curva de secado y (b) rapidez de deshidratación porcentual de la piña.

\subsection{COMERCIALIZACIÓN DE LOS SNACK SOLAR}

Como todo emprendimiento empresarial de un producto no conocido y con escaso capital, decidimos primeramente hacer conocer nuestro producto en nuestro ámbito universitario, para ello participamos en diferentes ferias de productos alimenticios o de generación de micro empresas, fue así que ganamos el

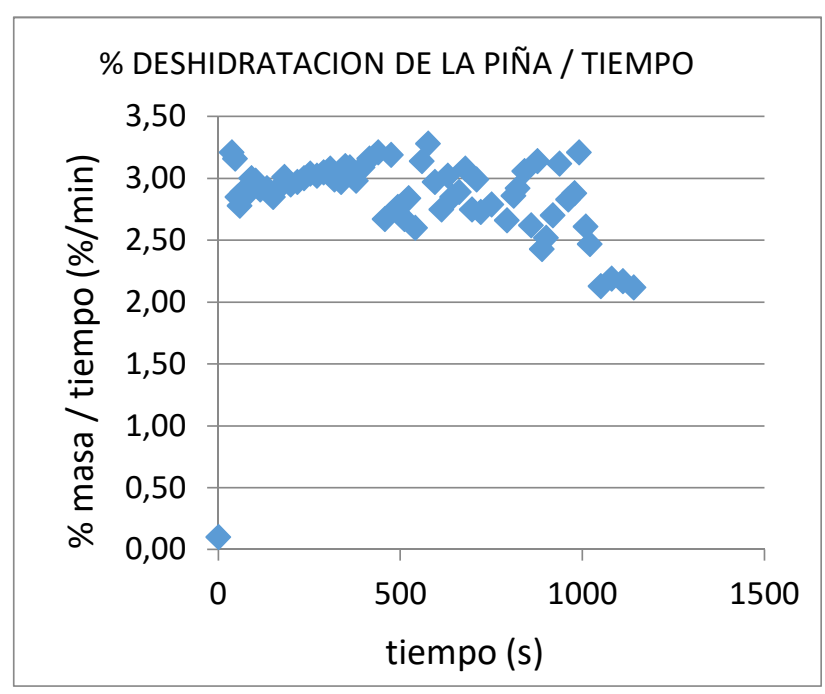

b)

Primer Puesto en la X Feria de Ciencia, Ingeniería y Tecnología Alimentaria (FECITAS - 2017) el cual nos permitió llevar nuestros Snack Solar al XII Congreso Nacional de Ciencia y Tecnología de Alimentos (CONACYTA) en la ciudad de Puno, teniendo una representación reconocida como producto innovador. 


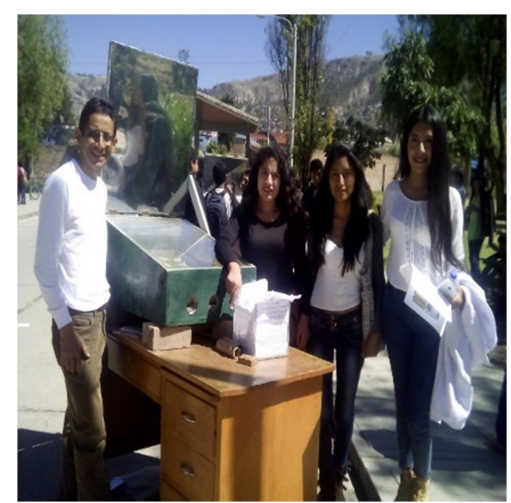

a)

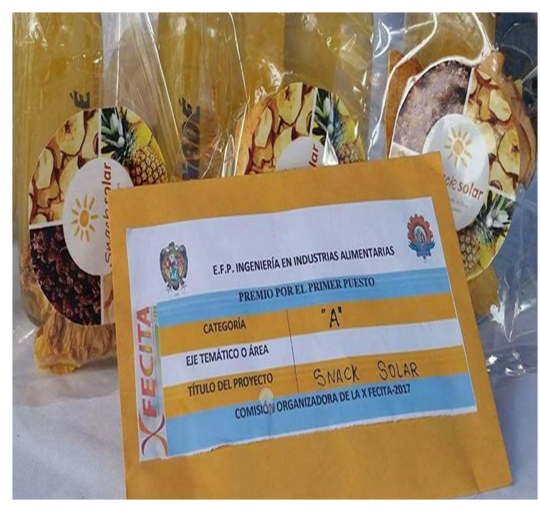

b)

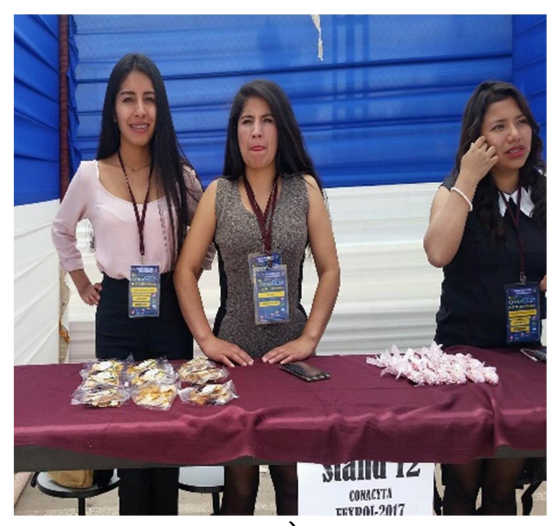

c)

Fig. 9. Difusión y comercialización de los Snack Solar. (a) En la UNSCH, (b) ganadores en FECITAS - 2017 y (c) en CONACYTA, Puno

A la fecha mantenemos en proyecto postular a concursos de financiamiento de incubadoras de empresas innovadoras a fin de aumentar nuestra capacidad de producción, siempre en la mano de los jóvenes voluntarios del CER UNSCH, gracias a quienes se pudo concretar este proyecto.

\section{CONCLUSIONES}

- El MSM es un módulo que integra, sobre la base de un colector solar plano, el uso independiente de un destilador, secador, terma y cocina solar, orientada como una iniciativa ambientalmente responsable, dentro del concepto de tecnologías apropiadas.

- El MSM- secador, es un secador pasivo de cabina con colector solar plano. Sin carga, la temperatura del aire a la salida de la cabina alcanza $74^{\circ} \mathrm{C}$, en este rango de temperaturas se pude secar casi todos los productos a baja escala.

- Cuando se reduce el contenido de agua de las frutas, aumenta la concentración de sus nutrientes, especialmente en este trabajo comprobamos que aumenta su concentración de ácido ascórbico (vitamina C), así en $100 \mathrm{~g}$ de manzana aumentó de $0.833 \mathrm{mg}$ a $13.33 \mathrm{mg}$, en 100 g de piña aumentó de $27.5 \mathrm{mg}$ a $39.33 \mathrm{mg}$ y en 100 g de plátanos aumento de $13.83 \mathrm{mg}$ a $39.33 \mathrm{mg}$.

- Este trabajo es una iniciativa de crear una microempresa utilizando la energía solar térmica, en un producto innovador, como es el consumo de frutas deshidratadas al sol.

\section{AGRADECIMIENTOS}

A las estudiantes de la Escuela Profesional de Ingeniería de Industrias Alimentarias de la UNSCH: Katherine Noa, Nataly Chávez, Daysi Mitma y Vanessa Quintana, por su apoyo y dedicación en este emprendimiento.

\section{REFERENCIAS}

[1] Centro de Conservación de Energía y del Ambiente (CERNERGIA), Diagnóstico de la situación actual del uso de la energía solar y eólica en el Perú, 2003.

[2] J. J. Hermosillo, Energía Solar, México: Instituto Tecnológico y de Estudios Superiores de Occidente, 1995.

[3] P. H. S. Santos y M. A. Silva, "Retention of Vitamin C in Drying Processes of Fruits and Vegetables", A Review, Drying Technology, vol. 12, no. 26, pp. 1421-1437, 2008.

[4] T. Blanco, Alimentos nativos del Perú al mundo. Lima: Editorial USIL, 2016.

[5] AOAC, Official methods of Analysis of the AOAC, vol 2, Arlington, USA: Association of Official Analytical Chemists Inc., 1990.

[6] K. Janampa et al., "Construcción y optimización de un módulo solar multipropósito para Ayacucho", Revista de Investigación de la UNSCH, 2009.

[7] I. Montero, "Modelado y construcción de un secador solar híbrido para residuos biomásicos", Tesis doctoral Badajoz, 2005.

[8] S. Nandwani, Energía solar y sus aplicaciones. Guácimo, Limón, Costa Rica: Universidad Earth, 2006.

[9]Instituto Nacional de Salud, Tablas peruanas de composición de alimentos ( $8^{\circ}$ ed.), Lima: Ministerio de Salud, Instituto Nacional de Salud, 2009.

[10] S. Baduí Dergal, Química de los Alimentos, México: Pearson Educación de México, 2013.

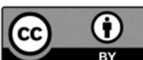
de este alcance pueden ser consultados a través del correo revistas@uni.edu.pe 